“ (C) 2017 IEEE. Personal use of this material is permitted. Permission from IEEE must be obtained for all other uses, in any current or future media, including

reprinting/republishing this material for advertising or promotional purposes, creating new collective works, for resale or redistribution to servers or lists, or reuse of any copyrighted component of this work in other works." 


\title{
Giant Enhancement and Control of Second-Harmonic Radiation from AlGaAs Nanoantennas
}

\author{
M. Rahmani ${ }^{1, *}$, S. Kruk ${ }^{1}$, R. Camacho-Morales ${ }^{1}$, L. Xu ${ }^{1}$, L. Wang ${ }^{1}$, A. E. Miroshnichenko ${ }^{1}$, D. Smirnova ${ }^{1}$, \\ H. Tan ${ }^{2}$, F. Karouta ${ }^{2}$, S. Naureen ${ }^{2}$, K. Vora ${ }^{2}$, A. S. Solntsev ${ }^{1}$, L. Carletti ${ }^{3}$, C. De Angelis ${ }^{3}$, C. Jagadish ${ }^{2}$, \\ Y. S. Kivshar ${ }^{1}$ and D. N. Neshev ${ }^{1}$ \\ ${ }^{1}$ Nonlinear Physics Centre, Research School of Physics and Engineering, \\ Australian National University, Canberra ACT 2601, Australia \\ 2 Department of Electronic Materials Engineering, Research School of Physics and Engineering, \\ Australian National University, Canberra ACT 2601, Australia \\ 3 \\ Department of Information Engineering, University of Brescia, 25123 Brescia, Italy \\ *mohsen.rahmani@anu.edu.au
}

\begin{abstract}
We fabricate AlGaAs nanoantennas on a glass substrate and demonstrate the highest nonlinear conversion efficiency of $10^{-4}$ with the capability for shaping the radiation patterns and polarization of the second harmonic emission in both forward and backward directions. We also decode dynamic multipolar contributions to the second harmonic generation within such nanoantennas.
\end{abstract}

Keywords- Subwavelength nanostructures; Nonlinear Spectroscopy; Semiconductors

\section{INTRODUCTION}

Nonlinear nanophotonic is at the heart of modern nanophotonics that aims toward the efficient light manipulation at the nanoscale and the design of ultrafast compact optical devices. This has led to many applications ranging from high performance data processing and optical computing to super imaging and biosensing. For a number of years, nonlinear nanophotonics studied metallic nanostructures with intrinsically high nonlinearity. However, metallic structures suffer from Ohmic losses at optical frequencies which limit their efficiency and functionalities. Alongside this, metals tend to have a relatively low heat resistance to high power lasers and this could be a key limiting factor to nonlinear optics at the nanoscale. This is why the observed nonlinear frequency conversion in plasmonic nanostructures remains small, of the order of $\sim 10^{-8}$, despite the implementation of different strategies to boost it, such as resonant coupling, threedimensional geometries, or hybrid nanoantennas $[1,2]$. On the other hand, dielectric and semiconductor nanomaterials can overcome these problems since their optical losses at visible and near-IR wavelengths are almost negligible. Such nanoparticles can support both electric and magnetic Mie-type resonances enabling multipolar and directional scattering of light by optical nanoantennas [3, 4]. This makes dielectric nanostructures a promising alternative for the frequency conversion at the nanoscale, due to the fact that a small volume of metamaterials and even isolated nanoantennas can create a surprisingly strong nonlinear effect $[5,6]$.

\section{RESULTS AND DISCUSSIONS}

An efficient SHG is obtained from a dielectric $\mathrm{Al}_{0.2} \mathrm{Ga}_{0.8} \mathrm{As}$ nanoantennas, in which nonlinear conversion efficiencies exceeding $10^{-4}$ can be achieved. Meanwhile, we demonstrate the possibility of shaping the second harmonic radiation pattern in forward and backward directions as well as its polarization state [7]. To realize this breakthrough, we implement a novel fabrication procedure of AlGaAs-in-insulator, containing epitaxial growth in conjunction with a bonding procedure to a glass substrate (see Fig. 1). It is a remarkable advantage to the previously fabricated III-V nanostructures on a GaAs handle wafer, which are limited to backward scattering, only [8, 9].

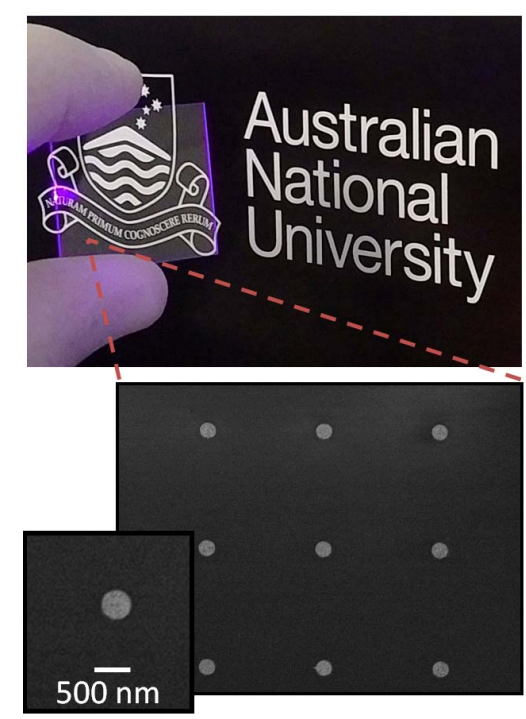

Fig. 1. Image of a glass substrate (top) supporting the fabricated AlGaAs nanostructures (bottom). 
Our AlGaAs nanodisks are designed to support Mie-type resonances at the wavelengths of both the fundamental wave and the second harmonic. The nanodisk AlGaAs antennas are excited by a pulsed femtosecond laser at wavelength of $1556 \mathrm{~nm}$ The laser beam of average beam power of $\sim 1 \mathrm{~mW}$ is then focused by an infrared objective $(\mathrm{NA}=0.85)$ to a diffraction limited spot of $2.2 \mu \mathrm{m}$, resulting in peak intensity of $\sim 7 \mathrm{GW} / \mathrm{cm} 2$. The configuration of our fabricated sample allows characterizing through the substrate both forward and backward SHG signals in the momentum (Fourier) space, using back focal planes (BFPs) imaging.
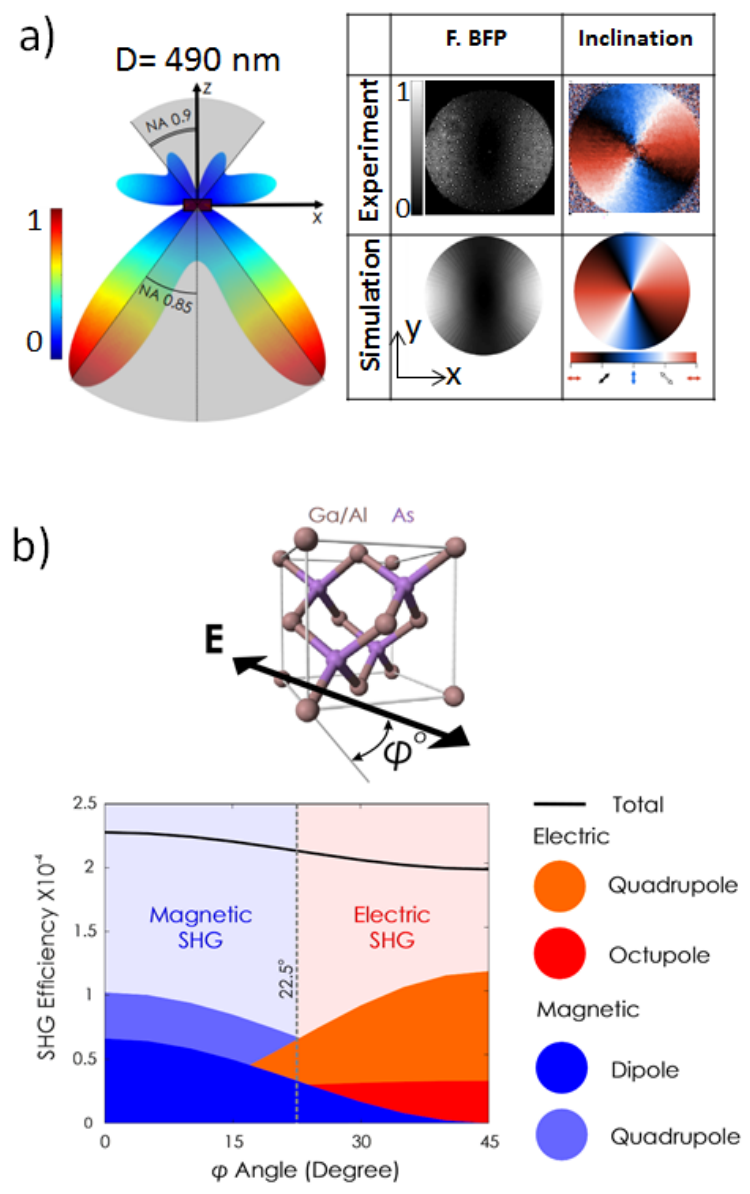

Fig. 2. (a) Front view of the far-field radiation pattern (left panel). Right panel: simulated and measured forward directionality diagrams (BFPs). (b) Dominant multipolar contributions to the second harmonic radiation by AlGaAs nanoantennas at different polarization angles, for the AlGaAs disk with the dimeter of $490 \mathrm{~nm}$.

Figure $2 \mathrm{a}$, left panel shows a $2 \mathrm{D}$ view of the calculated farfield radiation pattern of $\mathrm{SH}$ emission. Cones indicate range of angles experimentally accessible with our high-NA objectives. We use linear polarization of the pump, which is at $45^{\circ}$ to the crystalline axes of the AlGaAs nanodisks. As can be seen in the right panel, the forward back-focal plane (F. BFP) radiation pattern for the disk with diameter of $490 \mathrm{~nm}$ is in a good agreement with numerical simulations. Importantly, the measured radiation pattern exhibits vanishing SHG in the direction normal to the disks. This is reflected in the doughnut shape of BFP images due to the quadrupole $\mathrm{SH}$ radiation $[4,7]$. We also observe shaping of the polarization of the emitted $\mathrm{SH}$, where nearly-perfect radial polarization of the $\mathrm{SH}$ in experiment in forward direction is formed. These polarization states of the SH beam numerically, were also predicted by numerical calculations. Interestingly, we also found that for the polarization of pump at various angles to the AlGaAs crystalline axis, the second harmonic is dominated by the by a particular multipole. As can be seen in Fig. $2 \mathrm{~b}$, the $0^{\circ}$ polarization of pump, the second harmonic is produced mainly by magnetic multipoles, however for the polarization of pump at $45^{\circ}$ to the AlGaAs crystalline axis, the second harmonic is dominated by the electric multipoles [10]. This is an exciting observation showing the possibility of control the nature of SHG, not via a physical alteration, but by means of the polarization excitation, only.

In summary, we have studied experimentally, for the first time to our knowledge, both forward and backward SHG radiation patterns generated from $\mathrm{AlGaAs}$ disks fabricated on a thin glass substrate. Our results open a novel way for applications of subwavelength nanophotonics to nonlinear spectroscopy of chemical and biological species where nonlinear signals from nanostructures are highly sensitive to its surrounding environment such as biological substances. Alongside this, our results can open new avenues for nonlinear imaging, and in particular for design of efficient nonlinear holograms.

\section{ACKNOWLEDGMENT}

The authors acknowledge the support by the Australian Research Council and participation in the Erasmus Mundus NANOPHI project, contract number 2013 5659/002-001. The authors acknowledge the use of the Australian National Fabrication Facility (ANFF), the ACT Node.

\section{REFERENCES}

[1] D. Smirnova, and Y. S. Kivshar. "Multipolar nonlinear nanophotonics." Optica, vol. 3, pp. 1241-1255, 2016.

[2] S. Gennaro, M. Rahmani, V. Giannini, H. Aouani, T. Sidiropoulos, M. Navarro, S. A Maier, and Rupert F Oulton, "The interplay of symmetry and scattering phase in second harmonic generation from gold nanoantennas," Nano Lett., vol. 16, pp. 5278-5285, 2016.

[3] A. Kuznetsov, A. E. Miroshnichenko, M. L. Brongersma, Y. S. Kivshar, and B. Luk'yanchuk, "Optically resonant dielectric nanostructures," Science, vol. 354, pp. 2472, 2016.

[4] M. Caldarola, P. Albella, E. Cortés, M. Rahmani, T. Roschuk, G. Grinblat, R. F. Oulton, A. Bragas, and Stefan A. Maier, "Non-plasmonic nanoantennas for surface enhanced spectroscopies with ultra-low heat conversion," Nat. Commun., vol. 6, pp. 7915, 2015.

[5] G. Grinblat, M. Rahmani, E. Cortés, M. Caldarola, D. Comedi, S. A. Maier, and A. V. Bragas "High-efficiency second harmonic generation from a single hybrid $\mathrm{ZnO}$ nanowire/Au plasmonic nano-oligomer," Nano Lett., vol. 14, pp. 6660-6665, 2014.

[6] M. Shcherbakov, D. Neshev, B. Hopkins, A. Shorokhov, I. Staude, E. V. Melik-Gaykazyan, M. Decker, A. Ezhov, A. Miroshnichenko, I.Brener, A. Fedyanin, and Y. Kivshar, "Enhanced third-harmonic generation in silicon nanoparticles driven by magnetic response," Nano lett., vol. 14, pp. 6488-6492, 2014.

[7] R.. Camacho-Morales, M. Rahmani, S. Kruk, L. Wang, L. Xu, D.A. Smirnova, A. Solntsev, A. Miroshnichenko, H. Tan, F. Karouta, S. 
Naureen, K. Vora, L. Carletti, C. De Angelis, C. Jagadish, Y. S. Kivshar, and D. N. Neshev, "Nonlinear generation of vector beams from AlGaAs nanoantennas," Nano Lett., vol. 16, pp. 7191-7197, 2016.

[8] V. Gili, L.Carletti, A. Locatelli, D. Rocco, M. Finazzi, L. Ghirardini, I. Favero, C. Gomez, A. Lemaître, M. Celebrano, and C. De Angelis, "Monolithic AlGaAs second-harmonic nanoantennas," Opt. Exp., vol. 24, pp.15965-15971, 2016.

[9] L. Sheng, M. Sinclair, S. Saravi, G. A. Keeler, Y. Yang, J. Reno, G. M. Peake, f. Setzpfandt, I. Staude,T. Pertsch, and I. Brener, "Resonantly enhanced second-harmonic generation using III-V semiconductor alldielectric metasurfaces," Nano Lett., vol. 16, pp. 5426-32, 2016.

[10] S. Kruk, R. Camacho-Morales, L. Xu, M. Rahmani, D.A. Smirnova, L. Wang, H. H. Tan, C. Jagadish, D. N. Neshev, Y. S. Kivshar, "Nonlinear optical magnetism revealed by second-harmonic generation in nanoantennas," Nano Lett. vol. 17, 3914-3918, pp 2017. 